\section{Deletion of SPARC Enhances Retinal Vaso-Obliteration in Mouse Model of Oxygen- Induced Retinopathy}

\section{Doaa Sobeih ${ }^{1,2,3}$, Khaled A Hussein ${ }^{1,2,3}$, Neveen Said ${ }^{4}$, Kouros Mohamed $^{5}$ and Mohamed AI-Shabrawey ${ }^{1,2,3,6^{*}}$}

'Oral Biology/Anatomy, College of Dental Medicine, Georgia Regents University (GRU), Augusta, GA, USA

2James \& Jean Culver Vision Discovery Institute, GRU, USA

${ }^{3}$ Ophthalmology, Medical College of Georgia (MCG), GRU, USA

${ }^{4}$ Department of Radiation Oncology, University of Virginia School of Medicine, USA

${ }^{5}$ gdrasol, Irivin, CA, USA

${ }^{6}$ Cellular Biology and Anatomy, MCG, GRU, USA

\begin{abstract}
Background

Secreted Protein Acidic and Rich in Cysteine (SPARC) is a matricellular protein which is implicated in regulation of angiogenesis. Purpose

To characterize the changes in SPARC expression and effect of its deletion in a mouse model Oxygen Induced Retinopathy (OIR).

Materials and methods

Wild type (wt) and SPARC-deficient mice were subjected to high oxygen (75\%) for 5 days (p7-p12) before room air for additional 5 days (p12-p17). Retinas from both groups were flat mounted and retinal vessels were labeled with Isolectin-B4. Areas of Retinal Neovascularization (RNV) and vaso-obliteration were measured by Image-J and normalized to total retinal areas. SPARC expression was analyzed in both groups at p14 and p17 in retinal homogenates and sections by Western Blotting (WB) and immunofluorescence respectively. Human Retinal Endothelial Cells (HRECs) were exposed to hypoxia ( $1 \%$ O2) for 6 hours then SPARC was measured in cell lysate and condition medium by WB and ELISA. Moreover, HRECs were treated with VEGF or SPARC to study their mutual regulatory effect.
\end{abstract}

*Corresponding author: Mohamed Al-Shabrawey, Department of Oral Biology, College of Dental Medicine, College of Dental Medicine, 1120 15th Street, Augusta, GA 30912, USA, Tel: 7067212526; E-mail: malshabrawey@gru.edu

Citation: Sobeih D, Hussein KA, Said N, Mohamed K Al-Shabrawey M (2014) Deletion of SPARC Enhances Retinal Vaso-Obliteration in Mouse Model of Oxygen-Induced Retinopathy. J Ophthalmic Clin Res 1: 002.

Received: June 01, 2014; Accepted: August 08, 2014; Published: August 22, 2014

\begin{abstract}
Results
SPARC-deficient mice demonstrated significant increase in the vaso-obliteration ( $p=0.03$ ) and modest increase in RNV compared to the wt control. Retinal levels of SPARC was significantly decreased during OIR at $p 14(p=0.01)$ and partially restored to normal level by $\mathrm{p} 17$. Moreover, hypoxia significantly reduced SPARC expression and secretion in HRECs $(p=0.001)$. We noticed a mutual positive regulatory feedback between SPARC and VEGF.

\section{Conclusion}

SPARC deletion enhances ischemic retinopathy, thus modulation of SPARC expression could be a novel therapeutic approach to prevent pathological RNV.
\end{abstract}

\section{Introduction}

Pathological Retinal Neovascularization (RNV) is the main component of vision threatening retinal diseases [1] such as Proliferative Diabetic Retinopathy (PDR) [2], retinal vein occlusion, Retinopathy of Prematurity (ROP) [3], and Age related Macular Degeneration (AMD) [4]. The imbalance between the retinal levels of anti-angiogenic and pro-angiogenic factors e.g., Pigment Epithelial Derived growth Factor (PEDF) [5] and Vascular Endothelial Growth Factor (VEGF) [6,7] respectively in response to Retinal Hypoxia Leads to RNV [8]. Oxygen Induced Retinopathy (OIR) animal model is a well-established model of pathological RNV [9] that has significantly contributed to our understanding of the role of different factors involved in pathological RNV. Despite the fact that several factors were identified as contributors to the development of pathological RNV, VEGF is still considered the most important factor and this explains the revolutionary evolution of anti VEGF therapy to treat ocular NV [10].

It is well known that VEGF angiogenic effects occur through activation of VEGFR-2 (KDR); which is considered the main angiogenic receptor of VEGF. Meanwhile, the role of VEGFR-1(FLT1) is still unclear. Prior reports showed that it negatively affects the pathological NV and counteracts the mitogenic effect of VEGFR-2 $[11,12]$. Bussolati and his colleagues demonstrated that VEGFR-1 releases Nitric Oxide (NO) that acts as a molecular switch to limit the mitogenic activity of VEGFR-2 and promotes the differentiation of the cells into vascular tubes [13].

This was confirmed by earlier studies that showed that absence of VEGFR-1 is lethal to mice as a result of abnormal vascular development [14]. Moreover, studies by Rahimi et al., on pig aortic endothelial cells demonstrated that activation of VEGFR-1 halts angiogenesis by antagonizing VEGFR-2 responses [15]. On the other hand, recent studies showed that selective stimulation of VEGFR-1, using its specific agonist (Placenta Growth Factor-1; PlGF-1) was critical to vessel survival by preventing vaso-obliteration in retina during the hyperoxic stage of OIR (p7-p12) without affecting vaso-proliferation [16].

The Secreted Protein Acidic and Rich in Cysteine (SPARC) is a matricellular protein with contradictory role in angiogenesis. SPARC 
was heavily studied in tumor angiogenesis [17-19]. Several studies demonstrated SPARC as angiostatic molecule as a result of the negative correlation with VEGF expression in cancer colon [20], and glioma [18]. The regulatory role of SPARC in angiogenesis has been linked to both VEGF and Metalloproteinase (MMP) signaling. In particular, it has the ability to inhibit the tyrosine phosphorylation of FLT1 [21], regulating VEGF expression as well as the activity of MMP7 and 9 [22,23]. In contrast, SPARC over-expression was associated with increased angiogenesis in posterior uveal melanoma [24] and was found to be highly expressed in renal cell carcinoma, a highly vascular tumor overexpressing VEGF [25]. On the other hand, the role of SPARC in RNV has not been yet elucidated. Recently, SPARC overexpression by subretinal injection of recombinant adenovirus has been shown to induce preretinal NV and growth of new vessels in the inner plexiform layer in the rat eye. This was associated with a noticeable increase in the permeability and blood vessels proliferation with simultaneous overexpression of SPARC and VEGF when compared with VEGF overexpression [26]. On the other hand, inhibition of SPARC switch the angiogenic effect of VEGF-A to angiostatic effect and decreased the size of laser induced Choroidal $\mathrm{NV}(\mathrm{CNV})$, and this effect was inhibited after intravitreal injection of rhSPARC. Moreover, injection of anti-SPARC L-peptide the day before CNV laser induction was able to decrease the size of CNV [27]

Taken together previous reports about the regulatory role of SPARC in angiogenesis, we hypothesized that SPARC might be a crucial factor in regulating RNV. Thus, the aim of the current study was to characterize the changes in the levels of retinal SPARC in OIR mouse model and to test the effect of its deletion on RNV and vascular regeneration during OIR. We also investigated the effect of hypoxia on SPARC expression and the mutual regulatory effect between SPARC and VEGF in cultured Human Retinal Endothelial Cells (HRECs).

\section{Materials and Methods}

\section{Animals}

Wild type mice C57bl/6J and SPARC knockout mice were purchased from Jackson Laboratory (Bar Harbor, ME) and were used as a model of Oxygen Induced Retinopathy (OIR).

SPARC knockout mice were prepared and backcrossed with C57Bl/6 mice before use as described before $[28,29]$. The study was strictly adhered with the ARVO statement for the use of animals in ophthalmology and vision research and approved by animal care committee at Georgia Regents University. Both wild type and SPARC knockout OIR mouse model were prepared as we described previously $[9,30,31]$. Briefly, all newborn pups $(n=10)$ were kept at room air during the first 7 post natal days (p7) then they were transferred to high oxygen $(75 \%$ O2) chamber (Biospherix, Lacona, NY) for 5 days (p7 - p12) before they were returned to room air (oxygen 21\%). Sacrifice was carried out at p14 $(n=5)$ and p17 $(n=5)$. Age matching mice were kept at room air and used as control $(n=5)$. One retina of each animal was homogenized using RIPA buffer supplemented with protease and phosphatase inhibitor cocktail to be used for Western blot analysis and the other eye was processed as frozen sections or retinal flat-mount as previously described by us for immunofluorescence studies [30,31].

\section{Cell culture}

Human Retinal Endothelial Cells (HRECs) were purchased from Cell Systems Inc. (Kirkland, WA) and were grown in M199 media with 10\% Fetal Bovine Serum (FBS) and 1\% Bac-Off antibiotic (Cell Systems Inc. Kirkland, WA) in $37^{\circ} \mathrm{C}$ in humidified $95 \%$ air and $5 \%$ CO2. Cells were grown to $80-90 \%$ confluence on gelatin coated dishes then serum starved for 24 hours before starting the treatment and throughout the whole experiments.

To investigate the effect of SPARC on VEGF expression, HRECs were treated for 24 hours with or without different concentrations of SPARC $(100 \mathrm{ng} / \mathrm{ml}, 200 \mathrm{ng} / \mathrm{ml}$, and $300 \mathrm{ng} / \mathrm{ml})$. Meanwhile, to test the effect of VEGF on SPARC secretion, HRECs were treated with or without different concentrations of VEGF $(10 \mathrm{ng} / \mathrm{ml}, 50 \mathrm{ng} / \mathrm{ml}$, and $100 \mathrm{ng} / \mathrm{ml})$. At the end of the experiment the Conditioned Media (CM) was collected and analyzed by ELISA and the cells were homogenized in RIPA buffer then cell lysate was collected for Western blotting assay.

\section{Western blotting}

Western blotting analysis was performed on HRECs lysate and retina homogenates of OIR experiment to assess the expression of SPARC and VEGF proteins. Equal amount of protein lysates were separatedby $1 \%$ SDS-contained $10 \%$ PolyacrylamideGelElectrophoresis (SDS-PAGE) then transferred to PVDF membrane. The blot was blocked by $5 \%$ non-fat dry milk for one hour then incubated with 1/1000 rabbit anti- SPARC antibody (Cell Signaling, Carlsbad, CA) or 1/500 mouse anti-VEGF antibody (Abcam, Cambridge, MA). The loading control used was $\beta$-Actin detected using $1 / 500$ Goat polyclonal anti $\beta$-Actin antibody (Abcam, Cambridge, MA) diluted in blocking buffer. Incubation was done at $4^{\circ} \mathrm{C}$ overnight for SPARC and VEGF and at room temperature for 1 hour for $\beta$-Actin. After incubation of the membrane with the primary antibody rinsing was done for 5 times ( 5 minutes each) using $0.1 \%$ TBST to remove any residual antibodies. Then the membrane was incubated with the corresponding HRP-conjugated secondary antibodies (1:2000 in $0.1 \%$ TBST) for 1 hour. The detection kit used was ECL+ detection kit and the resultant X-ray film was automatically developed. Finally, quantification of the proteins of interest was done relative to $\beta$-Actin using Image J software.

\section{Immunofluorescence studies of retinal vessels and SPARC expression}

Preparation of retinal whole mount: To identify the impact of SPARC deletion on pathological RNV, we studied the changes in retinal vasculatures in retinal whole mount labeled with the vascular marker isolectin-B4(Vector Laboratories, Burlingame, CA) as described by us in our previous studies [30,31]. Briefly the eye balls were enucleated and fixed in $4 \%$ paraformaldehyde overnight. Circumferential cut was performed to remove the cornea then the lens was carefully removed and retina was gently removed from the eye cup. This was followed by incubation in Isolectin-B4 $(15 \mu \mathrm{g} / \mathrm{ml})$ overnight at $4 \mathrm{oC}$, then in normal goat serum (30\%) to block nonspecific reaction. Retina was then incubated with Texas red-conjugated Avidin D $(25 \mu \mathrm{g} / \mathrm{ml})$ (Vector Laboratories, Burlingame, CA) for 2 hours in room temperature to visualize the isolectin-B4 which bind to retinal vessels. The retina was then flat mounted on a slide and covered with a coverslip. Finally images were collected using the confocal microscopy (LSM 510, Carl Zeiss Inc.). For morphometric analysis of the capillary dropout area (vaso-obliteration area) and new capillary tufts, we used the Image-J. The capillary dropout area and the area of new tufts were normalized to the total area of each retina.

To determine the changes in the levels and localization of SPARC during OIR, we used immunofluorescence staining of retinal frozen 


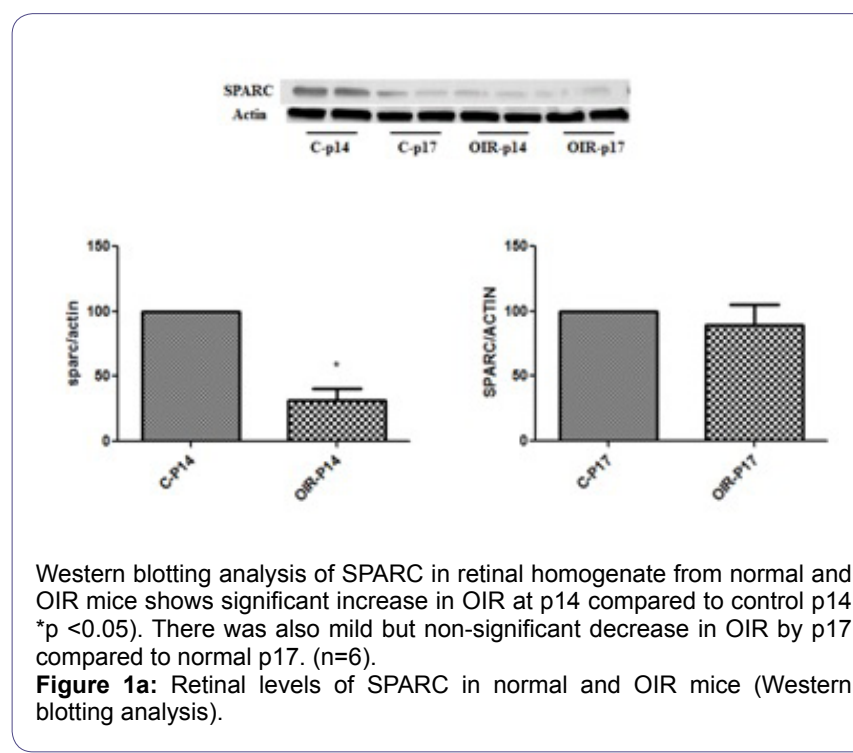

sections $(10 \mu$ thick). The expression of SPARC in mouse model of OIR and normal mice was detected by immunofluorescence using $1 / 200$ rabbit anti-SPARC antibody (Cell Signaling, Carlsbad, CA) and isolectin-B4 $(15 \mu \mathrm{g} / \mathrm{ml})$. Frozen sections were fixed in paraformaldehyde (4\%) for 10 minutes, washed with PBS (pH 7.5) 3 times/ 10 minutes, and permeabilized with Triton X (0.2\%) before they were incubated in normal goat serum $(30 \%)$ for 30 minutes. The sections were then incubated with anti SPARC primary antibody overnight in $4^{\circ} \mathrm{C}$. To Label the blood vessels and identify SPARC expression, sections then were washed with $1 \mathrm{x}$ PBS 3 times/ 10 minutes and TX $1 \%$ and incubated with $(25 \mu \mathrm{g} / \mathrm{ml})$ Texas red conjugated Avidin D (Vector Laboratories, Burlingame, CA) and 1/500 Oregon green-goat secondary anti-Rabbit (Life Technologies, Grand Island, NY) and protected from light for one hour at room temperature. Immuno-stained slides were covered using 4'6Diamidino-2-Phenylindole (DAPI) mounting medium (Vector Laboratories, Burlingame, CA) and images were obtained using Axiovision; Carl Zeiss Meditec, Inc., Dublin, CA.

\section{ELISA}

SPARC and VEGF secretion were determined in the conditioned media using a commercially available human SPARC and VEGF ELISA kit (R\&D Systems; MN, USA). The assay was performed according to the manufacturer's protocol.

\section{Statistical analysis}

To assure reproducibility experimental data were collected at least in triplicate except where noted. For the in vivo studies, we used at least 5 mice from each experimental group and for the in vitro studies 4 plates were used for each treatment and each experiment were repeated 3 times. The results are expressed as the mean $\pm \mathrm{SE}$. To compare 2 groups, the statistical analysis was performed using Student's paired t-test and ANOVA was used for multiple groups. A confidence level of $\mathrm{p}<0.05$ was considered statistically significant.

\section{Results}

Oxygen induced retinopathy is associated with low levels of

\section{SPARC}

To test whether SPARC is implicated in the development of ischemic retinopathy we examined the changes in its levels in retina of mouse model of OIR. Our experiments using Western blotting (Figure 1A) and immunofluorescence (Figure 1b) demonstrated significant decrease in retinallevels of SPARC during OIR in comparison to the control. This decline was statistically significant between animals during the early stage of the development of RNV in OIR at postnatal day 14 ( 2 days after relative hypoxia), $(\mathrm{p}=0.01)$ while it was not statistically significant for the animals at the postnatal day 17 (5 days after relative hypoxia).

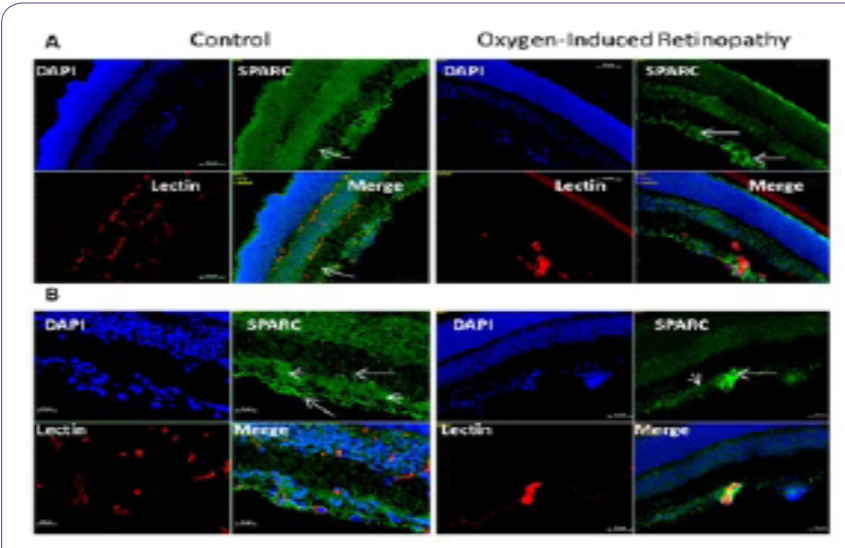

Immunofluorescence of SPARC expression in retina sections of control and OIR animal model at p14 shows marked decrease in SPARC immunoreactivity (green) in OIR. Note despite the SPARC is diffusely localized through different layer of normal retina, it is mainly localized in glial cells (arrows) and to less extent in ganglion cells (arrowhead) and inner nuclear layer. (blue stain: Nuclear marker DAPI, green stain SPARC, red stain vascular marker isolectin). A: 10x magnification, B: $20 \times$ magnification. Figure 1b: Retinal levels of SPARC in normal and OIR mice (Immunofluorescence analysis).

Immunofluorescence (Figure 1b) showed diffuse SPARC immunoreactivity in different layers of normal retina including nerve fiber, ganglion cells, inner and outer nuclear layers. The most noticeable immunoreactivity was localized in glial cell processes, perivascular in relation to the inner retinal vessels and in ganglion cells. On the other hand, SPARC immunoreactivity was much less in OIR compared to the control retina and was localized mainly in nerve fiber and ganglion cell layers, inner nuclear layer and RPE.

\section{Deletion of SPARC enhanced vaso-obliteration in OIR}

To further elucidate the potential role of SPARC in the development of RNV, we evaluated the impact of SPARC deletion on RNV in OIR model (Figure 2). SPARC deletion was associated with a significant increase in the area of vaso-obliteration compared to the wild type mice $(p=0.03)$. Increased retinal vaso-obliteration in SPARC-deficient mice was associated with mild but not significant increase in the RNV ( $\mathrm{p}>0.05)$.

\section{Hypoxia down-regulates SPARC expression and secretion}

\section{in HRECs}

Since hypoxia is crucial in mediating RNV, we evaluated the direct impact of hypoxia on SPARClevels in cultured HRECs. We tested SPARC protein levels in the cell lysate and CM of HRECs treated with or without $1 \%$ oxygen for 6-8 hours by Western blot and ELISA respectively (Figure 3). Hypoxia significantly reduced SPARC expression and secretion in comparison to the normoxia $(\mathrm{p}=0.001)$. These data are consistent with the changes in retinal levels of SPARC during the relative hypoxia in OIR model. 
Citation: Sobeih D, Hussein KA, Said N, Mohamed K Al-Shabrawey M (2014) Deletion of SPARC Enhances Retinal Vaso-Obliteration in Mouse Model of Oxygen-Induced Retinopathy. J Ophthalmic Clin Res 1: 002.

\section{VEGF induces SPARC secretion in HRECs}

The cross talk between SPARC and VEGF signaling has been investigated in different models of angiogenesis. Therefore, we were interested in finding out whether there is relation between VEGF and SPARC in retinal endothelial cells (Figure 4). The protein levels of SPARC in HRECs CM were significantly up-regulated $(\mathrm{p}<0.05)$ by various concentration of VEGF $(10,50$ and $100 \mathrm{ng} / \mathrm{ml})$. The maximum effect was noticed in cells treated with $100 \mathrm{ng} / \mathrm{ml}$.
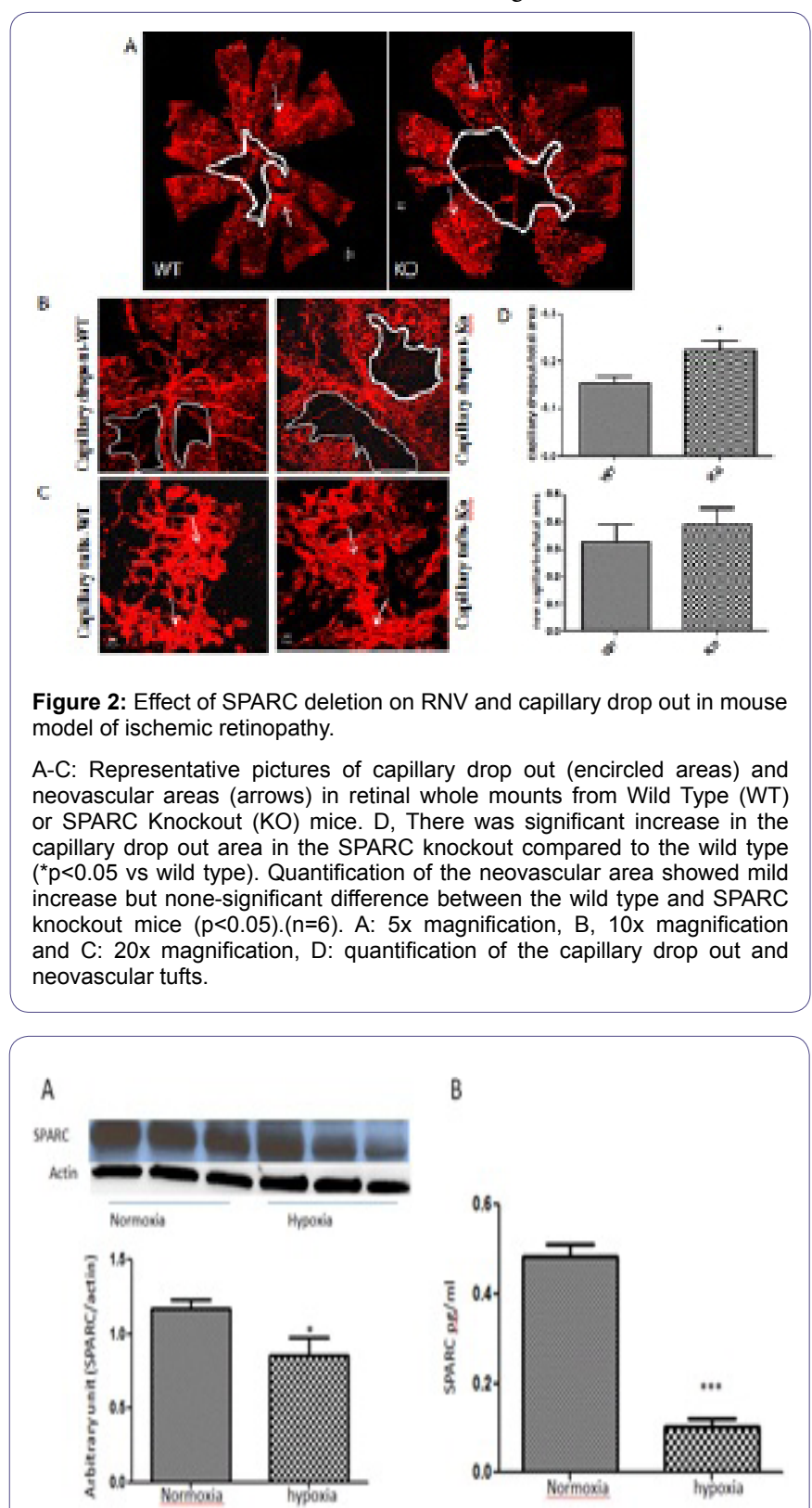

Figure 3: Effect of hypoxia on SPARC expression/secretion in HRECs.

(A) Western blotting analysis of SPARC expression in HRECs treated with or without hypoxia (1\% oxygen) for $6-8$ hours before collecting the cell lysate after 24 hours from the beginning of the experiment. Note, the marked decrease in SPARC expression under hypoxic condition ( $p<0.05$ vs normoxia).

(B) Similarly ELISA assay (B) of SPARC secretion in HRECs condition media demonstrated significant decrease in SPARC secretion by HRECs treated with hypoxia ( $p<0.0003$ vs normoxia) $n=3$.

\section{SPARC up-regulates VEGF expression in HRECs}

To further investigate the cross talk between SPARC and VEGF signaling in HRECs we also tested the impact of various concentration of SPARC treatment $(100,200$ and $300 \mathrm{ng} / \mathrm{ml})$ on VEGF protein expression by Western blotting. Our experiment demonstrated significant increase in VEGF expression in a concentration dependent pattern (Figure 5). Taken together data from (Figures 4,5), there is positive feedback between VEGF and SPARC.

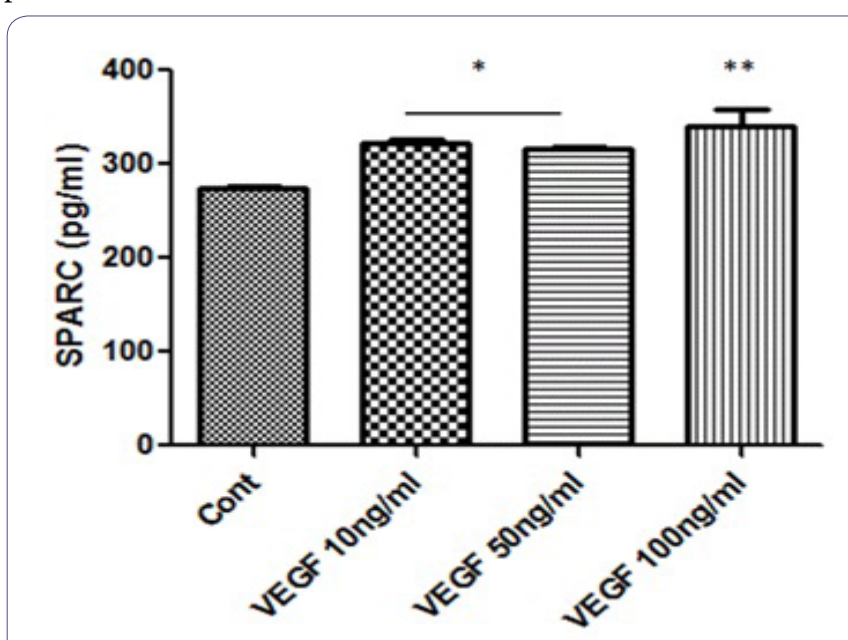

Figure 4: Effect of VEGF treatment on SPARC production in HRECs.

ELISA assessment of SPARC secretion in condition medium of HRECs treated with or without various concentrations of VEGF $(10,50$ and 100ng/ $\mathrm{ml})$ shows a concentration dependent increase in SPARC secretion. $\left({ }^{*} p<0.05\right.$ vs control, ${ }^{* *} p<0.002$ vs control) $n=5$.

\section{Discussion}

Although the role of SPARC in angiogenesis has been investigated in various disease models, its role in RNV has not yet elucidated. To the best of our knowledge the current study is the first to characterize the changes in the levels of SPARC in retina during pathological RNV in OIR and to determine the effect of its deletion on the development

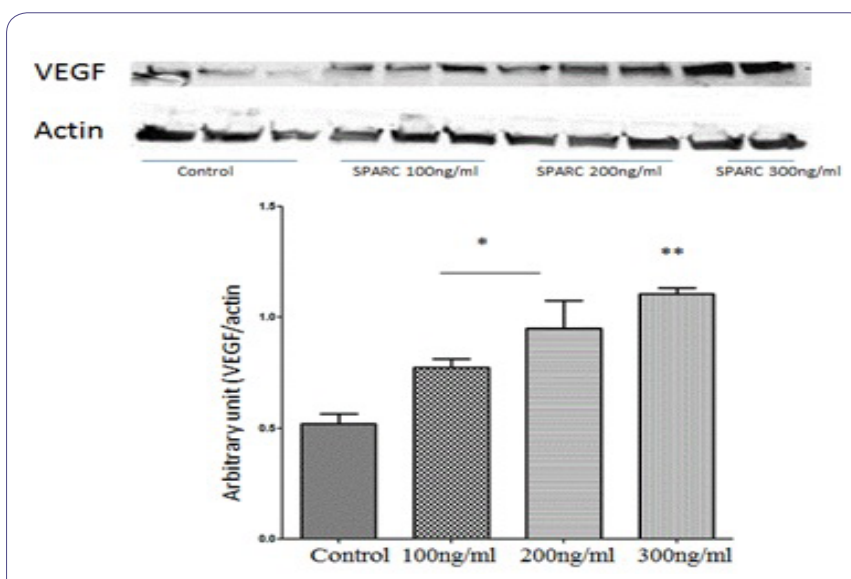

Figure 5: Effect of SPARC on VEGF production in HRECs.

Western blotting analysis of protein levels of VEGF in HRECs treated with or without various concentration of SPARC $(100,200$ and $300 \mathrm{ng} / \mathrm{ml})$. Note the concentration dependent increase in VEGF production by SPARC. The highest VEGF levels were noticed in HRECs treated with $300 \mathrm{ng} / \mathrm{ml}$. * $p<0.05$ vs control, ${ }^{* *} p<0.002$ vs control) $n=5$. 
of vaso-obliteration and RNV. The main findings of this study were: -Significant decrease in retinal levels of SPARC protein during early stage of OIR (p14),

-Enhanced vaso-obliteration and mild increase in RNV in SPARC-deficient mice during OIR,

- Down-regulation of SPARC production in HRECs by hypoxia, -Positive feedback between SPARC and VEGF treatment in HRECs.

A
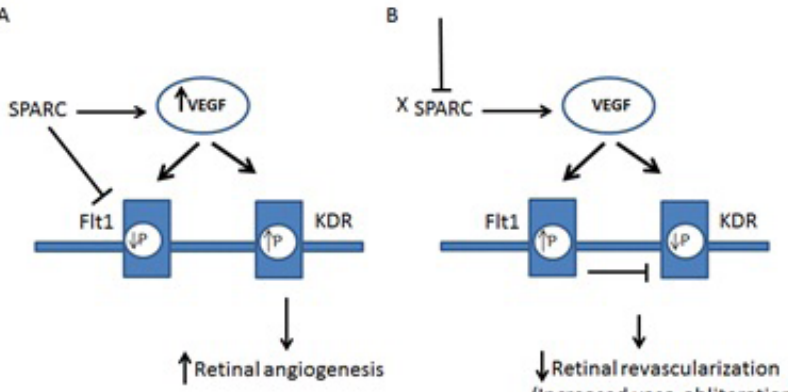

Figure 6: Schematic diagram showing suggested pathway for how deletion or the decrease of SPARC halts retinal revascularization and increases in OIR.

A) SPARC inhibits tyrosine Phosphorylation (P) of Flt1 switching the VEGF signaling to KDR leading to retinal angiogenesis. SPARC also increases VEGF expression. B) Deletion or decrease of SPARC leads to sustained activity of Flt 1 which negatively impacts the mitogenic activity of KDR and in turn attenuates revascularization of central retina causing increased capillary dropout area (vaso-obliteration).

SPARC is a matricellular protein that has a regulatory role in angiogenesis via modulating VEGF signaling which includes but not limited to the inhibition of tyrosine phosphorylation of VEGFR-1 [21]. VEGFR-1 (FLT1) was found to limit the angiogenic effect of VEGF by inhibiting the mitogenic activity of VEGFR-2 [15]. This might provide an explanation to our data that revealed failure of central retinal capillary regeneration in SPARC-deficient mice when compared to the wild type OIR. Although VEGF levels significantly increases in retina during OIR, it seems that absence of SPARC eliminated its inhibitory effect onVEGFR-1 causing suppression of the angiogenic activity of VEGFR-2. Our results are consistent with previous studies on laser-induced $\mathrm{CNV}$ in which inhibition of SPARC by anti- SPRAC oligopeptide was able to switch the effect of VEGF-A from being angiogenic to be anti-angiogenic via Flt-1 dependent mechanism [27]. Thus, SPARC drives the angiogenesis by inhibiting Flt 1 and subsequent activation of KDR by VEGF. Moreover, prior studies performed on SPARC knockout mice showed decrease in the size of laser-induced CNV after VEGF-A injection [32]. The modest increase in the pathological NV in OIR SPARC-deficient mice compared to wild type OIR could be attributed to compensatory new vessel formation due to increased hypoxia that results from increased vaso-obliteration in central retina.

Hypoxia treatment of HRECs showed significant reduction in the levels of SPARC expression and secretion. Whether this decrease in SPARC levels by hypoxia is required for endothelial survival via maintaining constitutively active VEGFR-1 needs further investigation.

Our data from wild type OIR animal model showed significant decrease in SPARC level by p14 which was restored back to normal levels by $\mathrm{p} 17$. The decrease in SPARC during the early stage of relative hypoxia ( $\mathrm{p} 14$ ) in OIR may contribute to the persistence of the central retinal capillary degeneration (vaso-obliteration) via switching VEGF signaling to VEGFR-1. However restoration of SPARC normal levels by p17 may enhance the angiogenic effect of VEGF retinal pool through suppression of VEGFR-1 tyrosine phosphorylation and thus switching VEGF signaling to VEGFR-2 mitogenic activity. In support of our data, overexpression of SPARC in rat's eye was able to induce subretinal NV by itself and aggravated the inflammatory and angiogenic effect of VEGF when combined overexpression of VEGF and SPARC [26]. Our in vitro studies performed on HRECs revealed that VEGF elicits regulatory effect on retinal SPARC. VEGF increased SPARC expression/secretion in HRECs suggesting that VEGF might induce its angiogenic effect in part through up-regulation of SPARC to suppress VEGFR-1 phosphorylation and subsequently eliminate its suppressive effect on VEGFR-2 [15]. Our in vitro data are supported by an earlier report that showed increased SPARC secretion by VEGF treatment in cultured Human Umbilical Vein Endothelial Cells (HUVECs). On the other hand, SPARC promoted VEGF expression in HRECs. Our in vitro data demonstrated positive feedback between the VEGF and SPARC treatment in HRECs suggesting that SPARC might play apro-angiogenic role not only through modulating the VEGFR-1 activity but also via regulating VEGF expression.

In conclusion, our data suggest that decreased SPARC production during OIR and in response to hypoxia might play a role in the development of RNV via enhancing central retinal vaso-obliteration. The underlying mechanism needs further investigation. However the regulatory role of SPARC on VEGF signaling through inhibition of VEGFR-1 tyrosine phosphorylation and regulation of VEGF expression might provide a clue. Taken together our current data and previous reports, we conclude that decreased SPARC production in wild type mice during OIR and lack of SPARC in the knockout mice enhances the activity of VEGFR-1 causing attenuation of vascular regeneration in the central retina during OIR (Figure 6). This also might explain the mild increase in RNV in SPARC-deficient mice which could be linked to the increased vaso-obliteration area and subsequent hypoxia and further increase in VEGF expression in the retina of this animal model. Our in vivo studies were generated from OIR animal model which mimic the retinopathy of prematurity in humans so further studies are needed to investigate whether the SPARC deletion and expression demonstrate similar pattern in other models of ocular neovasculrization. Our data suggest SPARC as a novel therapeutic target to prevent development of RNV during ischemic retinopathy. The use of SPARC during early stage of ischemic retinopathy might be beneficial in preventing capillary degeneration, the driving force for VEGF signaling and subsequent RNV during ischemic retinopathy via switching the activity of VEGF signaling to be mainly through VEGFR-2.

\section{Acknowledgment}

National Eye Institute 5R01EY023315-02 and Qatar National Research Fund NPRP 4-1046-3-284 to Mohamed Al-Shabrawey.

\section{References}

1. Miller JW, Le Couter J, Strauss EC, Ferrara N (2013) Vascular endothelial growth factor a in intraocular vascular disease. Ophthalmology 120: 106-114.

2. Klein BE (2007) Overview of epidemiologic studies of diabetic retinopathy. Ophthalmic Epidemiol 14: 179-183.

3. Sapieha P, Joyal JS, Rivera JC, Kermorvant-Duchemin E, Sennlaub F, et al. (2010) Retinopathy of prematurity: understanding ischemic retinal vasculopathies at an extreme of life. J Clin Invest 120: 3022-3032. 
4. Yannuzzi LA, Negrao S, lida T, Carvalho C, Rodriguez-Coleman $\mathrm{H}$, et al. (2012) Retinal angiomatous proliferation in age-related macular degeneration. 2001. Retina 1: 416-434.

5. Haurigot V, Villacampa P, Ribera A, Bosch A, Ramos D, et al. (2012) Longterm retinal PEDF overexpression prevents neovascularization in a murine adult model of retinopathy. PLoS One 7: 41511.

6. Achen MG, Stacker SA (1998) The vascular endothelial growth factor family; proteins which guide the development of the vasculature. Int J Exp Pathol 79: 255-265.

7. Aiello LP, Northrup JM, Keyt BA, Takagi H, Iwamoto MA (1995) Hypoxic regulation of vascular endothelial growth factor in retinal cells. Arch Ophthalmol 113: $1538-1544$

8. Gariano RF, Gardner TW (2005) Retinal angiogenesis in development and disease. Nature 438: 960-966.

9. Smith LE, Wesolowski E, McLellan A, Kostyk SK, D'Amato R, et al. (1994) Oxygen-induced retinopathy in the mouse. Invest Ophthalmol Vis Sci 35: 101-111.

10. Aiello LP, Pierce EA, Foley ED, Takagi H, Chen H, et al. (1995) Suppression of retinal neovascularization in vivo by inhibition of Vascular Endothelia Growth Factor (VEGF) using soluble VEGF-receptor chimeric proteins. Proc Natl Acad Sci USA 92:10457-10461.

11. Waltenberger J, Claesson-Welsh L, Siegbahn A, Shibuya M, Heldin $\mathrm{CH}$ (1994) Different signal transduction properties of KDR and Flt1, two receptors for vascular endothelial growth factor. J Biol Chem 269: 26988-26995.

12. Dvorak HF (2002) Vascular permeability factor/vascular endothelial growth factor: A critical cytokine in tumor angiogenesis and a potential target for diagnosis and therapy. J Clin Oncol 20: 4368-4380.

13. Bussolati B Dunk C, Grohman M, Kontos CD, Mason J, et al. (2001) Vascular endothelial growth factor receptor-1 modulates vascular endothelial growth factor-mediated angiogenesis via nitric oxide. Am J Pathol 159: 993-1008.

14. Fong GH, Zhang L, Bryce DM, Peng J (1999) Increased hemangioblast commitment, not vascular disorganization, is the primary defect in flt-1 knock-out mice. Development 126: 3015-3025

15. Rahimi N, Dayanir V, Lashkari K (2000) Receptor chimeras indicate that the Vascular Endothelial Growth Factor Receptor-1 (VEGFR-1) modulates mitogenic activity of VEGFR-2 in endothelial cells. J Biol Chem 275: 6986-16992.

16. Shih SC, Ju M, Liu N, Smith LE (2003) Selective stimulation of VEGFR-1 prevents oxygen-induced retinal vascular degeneration in retinopathy of prematurity. J Clin Invest 112: 50-57.

17. Gorantla B, Bhoopathi P, Chetty C, Gogineni VR, Sailaja GS, et al. (2013) Notch signaling regulates tumor-induced angiogenesis in SPARC-overexpressed neuroblastoma. Angiogenesis 16: 85-100.

18. Yunker CK, Golembieski W, Lemke N, Schultz CR, Cazacu S, et al. (2008) SPARC-induced increase in glioma matrix and decrease in vascularity are associated with reduced VEGF expression and secretion. Int J Cancer 122: 2735-2743.
19. Rocnik EF, Liu P, Sato K, Walsh K, Vaziri C (2006) The novel SPARC family member SMOC-2 potentiates angiogenic growth factor activity. J Biol Chem 281: 22855-22864.

20. Liang JF, Wang HK, Xiao H, Li N, Cheng CX, et al. (2010) Relationship and prognostic significance of SPARC and VEGF protein expression in colon cancer. J Exp Clin Cancer Res 29: 71.

21. Kupprion CK, Motamed, Sage EH (1998) SPARC (BM-40, osteonectin) inhibits the mitogenic effect of vascular endothelial growth factor on microvascular endothelial cells. J Biol Chem 273: 29635-29640.

22. Bhoopathi $P$, Chetty C, Gujrati M, Dinh DH, Rao JS, et al. (2010) The role of MMP-9 in the anti-angiogenic effect of secreted protein acidic and rich in cysteine. Br J Cancer 102: 530-540.

23. Zhang JL, Chen GW, Liu YC, Wang PY, Wang X, et al. (2012) Secreted protein acidic and rich in cysteine (SPARC) suppresses angiogenesis by down-regulating the expression of VEGF and MMP-7 in gastric cancer. PLoS One 7: 44618 .

24. Ordonez JL, Paraoan L, Hiscott P, Gray D, García-Fiñana M, et al. (2005) Differential expression of angioregulatory matricellular proteins in posterior uveal melanoma. Melanoma Res 15: 495-502.

25. Sakai N, Baba M, Nagasima Y, Kato Y, Hirai K, et al. (2001) SPARC expression in primary human renal cell carcinoma: upregulation of SPARC in sarcomatoid renal carcinoma. Hum Pathol 32: 1064-1070.

26. Watanabe K, Okamoto F, Yokoo T, lida KT, Suzuki H, et al. (2009) SPARC is a major secretory gene expressed and involved in the development of proliferative diabetic retinopathy. $\mathrm{J}$ Atheroscler Thromb 16: 69-76.

27. Uehara H, Luo L, Simonis J, Singh N, Taylor EW, et al. (2010) Anti-SPARC oligopeptide inhibits laser-induced CNV in mice. Vision Res 50: 674-679.

28. Said N, Frierson HF, Sanchez-Carbayo M, Brekken RA, Theodorescu D (2013) Loss of SPARC in bladder cancer enhances carcinogenesis and progression. J Clin Invest 123: 751-766.

29. Norose K, Clark JI, Syed NA, Basu A, Heber-Katz E, et al. (1998) SPARC deficiency leads to early-onset cataractogenesis. Invest Ophthalmol Vis Sci 39: $2674-2680$

30. Al-Shabrawey M, Bartoli M, El-Remessy AB, Platt DH, Matragoon S, et al. (2005) Inhibition of $\mathrm{NAD}(\mathrm{P}) \mathrm{H}$ oxidase activity blocks vascular endothelial growth factor overexpression and neovascularization during ischemic retinopathy. Am J Pathol 167: 599-607.

31. Al-Shabrawey M, Mussell R, Kahook K, Tawfik A, Eladl M, et al. (2011) In creased expression and activity of 12-lipoxygenase in oxygen-induced ischemic retinopathy and proliferative diabetic retinopathy: implications in retinal neovascularization. Diabetes 60: 614-624.

32. Nozaki M, Sakurai E, Raisler BJ, Baffi JZ, Witta J, et al. (2006) Loss of SPARC-mediated VEGFR-1 suppression after injury reveals a novel antiangiogenic activity of VEGF-A. J Clin Invest 116: 422-429. 